\title{
CONTROL OF SELF-TRANSFER-TYPE AUTOMATIC POURING ROBOT WITH CYLINDRICAL LADLE
}

\author{
Yoshiyuki Noda* Ken'ichi Yano** \\ Kazuhiko Terashima* \\ * Dept. of Production Systems Engineering, \\ Toyohashi University of Technology, \\ 1-1 Hibarigaoka, Tempaku, Toyohashi 441-8580 Japan \\ ** Dept. of Mechanical and Systems Engineering, \\ Gifu University, 1-1 Yanagido, Gifu 501-1193, Japan
}

\begin{abstract}
This paper is concerned with advanced control of self-transfer-type automatic pouring robot, with focus on the pouring control to cylindrical ladle and sloshing suppression (liquid vibration) control to the liquid in a ladle. In the pouring control, the flow rate model of cylindrical ladle is represented by LPV(Linear Parameter Varying) model, and FeedForward(FF) controller is then designed by using inverse systems of motor and flow rate model. In the sloshing suppression control, the natural frequency of sloshing of liquid in the ladle is varied by tilting ladle. Therefore, the natural frequency of sloshing is identified by using Short Time Fourier Transform, and the controllers are designed by Hybrid Shape Approach. The validity of proposed control system is demonstrated through experiments. Copyright $\left.{ }^{(}\right) 2005$ IFAC
\end{abstract}

Keywords: Automatic Pouring Robot, Pouring Control, Sloshing Suppression

\section{INTRODUCTION}

Automatic pouring robots have recently been applied to the pouring process in the casting industry. In these robots, the mold line is stopped at a fixed position, where the molten metal is poured from the ladle into the mold. After the pouring, the mold line is transferred again. The next mold is moved forward at the front position of the ladle and the mold line stops again. In this process, sand molds are sometimes destroyed by unsuitable acceleration of the line. In order to solve these problems, a self-transfer-type automatic pouring robot, such as that which synchronizes the movement of the ladle to the movement of the mold line (see Fig. 1), is much needed. However, this robot has the problem that sloshing of the molten metal in the ladle occurs when the ladle transfer accelerates. This sloshing is generated by inappropriate transfer control. The sloshing causes the overflow of molten metal from the ladle and leads to contamination, which degrades the quality of the metal. Therefore, it is important to build a pouring robot that makes the transfer of a ladle track to the mold line with sloshing suppression.

Moreover, there is another demand to automatic pouring robot. The fan-type ladle has been used to most of pouring robots. Because, in the pouring motion, the flow rate from the ladle keeps constant for the constant angular velocity of tilting ladle, and thus, it is easy to pour the molten metal into the mold. However, decreasing temperature of molten metal in the fan-type ladle is lower than that of cylindrical ladle. The quality of casting product is degraded by decreasing temperature. 


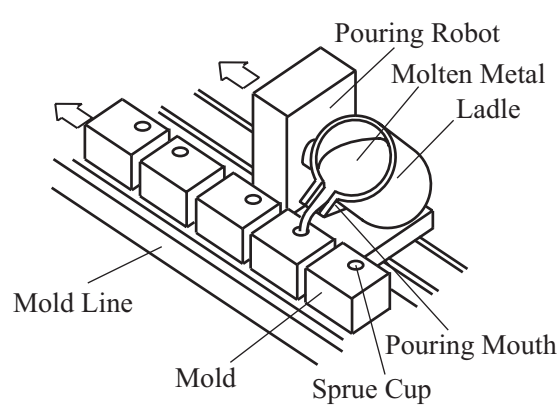

Fig. 1. Automatic pouring robot in a continuously moving mold line

Therefore, the development of pouring control in automatic pouring robot using cylindrical ladle has been demanded in the industrial plants.

In previous study, sloshing suppression control of automatic pouring robot designed by using Hybrid Shape Approach has been published(K. Yano and K. Terashima, 2001). The study is to develop the fixed-mold-type automatic pouring robot, which the molten metal is poured into the mold at the fixed position, with considering sloshing suppression. Also, the tracking control to moving object with considering sloshing suppression has been reported by authors(Y. Noda and K. Terashima, 2003). By using 2DOF control system, tracking and sloshing suppression controls have been achieved. However, the pouring motion is not considered in this system. Therefore, self-transfertype automatic pouring robot with considering sloshing suppression has not been published.

Studies on pouring control in the batch process treated in this paper have been published a little. The liquid level control in a sprue cup for the batch-type pouring processes by means of 2DOF control is reported(Y. Sugimoto and K. Terashima, 2002). However, above study is the pouring control for automatic pouring robot in the case of fan-type ladle. Therefore, this paper presents a new result of pouring and sloshing suppression control for self-transfer-type automatic pouring robot with cylindrical ladle.

\section{AUTOMATIC POURING ROBOT}

The laboratory experimental apparatus used in this paper is shown in Fig. 2. The automatic pouring robot can transfer the ladle on three di-

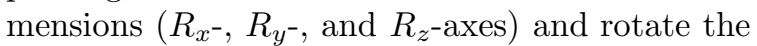
ladle $\left(R_{\theta}\right.$-axis). The mold line can transfer molds on $M$-axis. The rotary direction of the $R_{\theta}$-axis is driven by an $\mathrm{AC}$ servomotor. The driving force of the AC servomotor can be amplified by reducing the gear ratio. The center of the ladle's rotation shaft is placed near the ladle's center of gravity. When the ladle is rotated around the center of gravity, the tip of the pouring mouth moves in a circular trajectory. It is then difficult to pour the

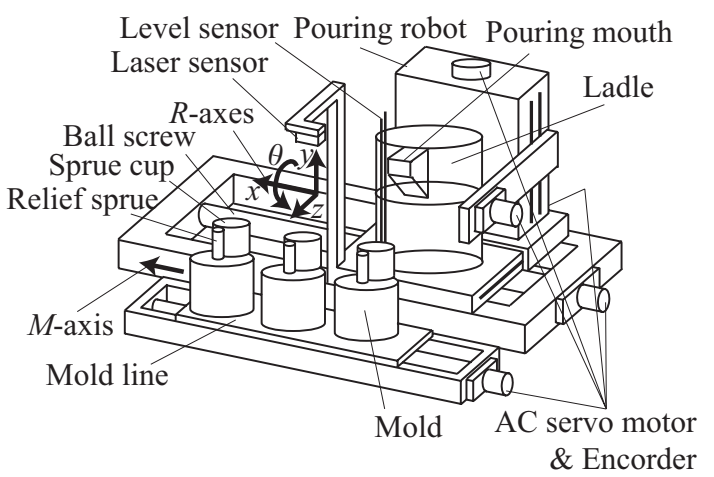

Fig. 2. Illustration of Automatic Pouring Robot

molten metal into a mold if the pouring mouth is moved by tilting. Then, the position of the tip of the pouring mouth is controlled invariable during pouring, by means of a synchronous control of $R_{y^{-}}$ and $R_{z}$-axes for rotational motion around $R_{\theta}$-axis of a ladle. The rotation angle is measured by an encoder installed in AC servomotor. $R_{x^{-}}, R_{y^{-}}$, and $R_{z}$-axes in automatic pouring robot and $M$-axis in mold line are also driven by $\mathrm{AC}$ servomotors. But, the driving force of each of these motors is amplified through the ball and screw mechanism. Each axis can be independently moved. The object liquid in the present experiments is water, whose static liquid level $h_{s}$ is $0.16[\mathrm{~m}]$. The liquid level in a sprue cup is measured by a laser sensor above the relief sprue. The laser sensor used in this paper can measure liquid levels higher than $0.036[\mathrm{~m}]$ from the bottom of a cup $(0[\mathrm{~m}])$. In order to measure the sloshing in the ladle, the liquid level is measured by using an electric resistancetype sensor comprised of two stainless bars at the position of the edge of the ladle.

\section{MODELING OF THE POURING PROCESS}

In this section, a modeling for the pouring process is carried out using water. The kinematical viscosity of the water $(293[\mathrm{~K}])$ and the molten metal $(1673[\mathrm{~K}])$ are $1.004 \times 10^{-6}\left[\mathrm{~m}^{2} / \mathrm{s}\right]$ and $0.970 \times 10^{-6}\left[\mathrm{~m}^{2} / \mathrm{s}\right]$, respectively. Therefore, the fluid behavior of the water is nearly equals to that of the molten metal. A schematic diagram of the pouring model is shown in Fig. 3.

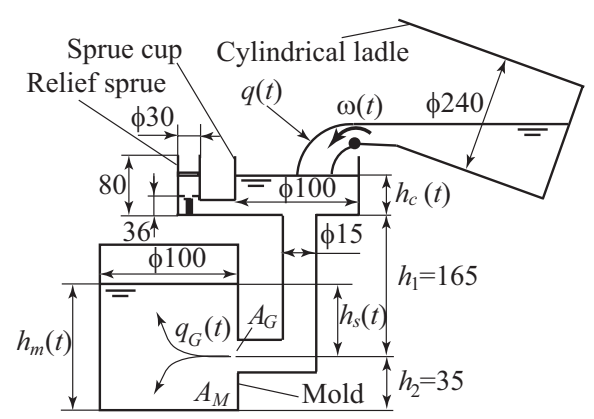

Fig. 3. Illustration of a pouring model 
Here, three models, from input voltage $u_{t}(t)[\mathrm{V}]$ for motor to the liquid level $h_{c}(t)[\mathrm{m}]$ in a sprue cup, are divided into three parts as follows.

(1) A model between input voltage of the AC servomotor $u_{t}(t)$ and the tilting angler velocity of a ladle $\omega(t): P_{m t}(s)$;

(2) A model between the tilting angler velocity of the ladle $\omega(t)$ and the flow rate into a sprue $\operatorname{cup} q(t): P_{f}(s)$;

(3) A model between the flow rate into the sprue cup $q(t)$ and the liquid level in a sprue cup $h_{c}(t): P_{h}(s)$;

$P_{m}(s), P_{f}(s)$, and $P_{h}(s)$ are called, respectively, motor model, flow rate model, and level model.

The relationship between the tilting angular velocity of the ladle and the input voltage to the motor is described in the following equation:

$$
\frac{d \omega(t)}{d t}=-\frac{1}{T_{t m}} \omega(t)+\frac{K_{t m}}{T_{t m}} u_{t}(t)
$$

where $\omega[\mathrm{rad} / \mathrm{s}]$ is the tilting angular velocity, $u_{t}(t)[\mathrm{V}]$ is the input voltage for $R_{\theta}$-axis, $T_{t m}[\mathrm{~s}]$ is the time constant of the motor, $K_{t m}[\mathrm{deg} / \mathrm{sV}]$ is the gain of the motor. Further, the motor models of the $R_{x^{-}}, R_{y^{-}}, R_{z^{-}}$, and $M$-axes are described in the same form as in Eq. (1), and the motor parameters are shown in Table 3 .

Table 1. Motor parameter

\begin{tabular}{lll}
\hline Parameter & Symbol & Value \\
\hline$R_{x}$-axis gain & $K_{x m}$ & $0.166[\mathrm{~m} / \mathrm{sV}]$ \\
\hline$R_{x}$-axis time constant & $T_{x m}$ & $0.007[\mathrm{~s}]$ \\
\hline$R_{x}$-axis max velocity & $V_{x \max }$ & $0.8[\mathrm{~m} / \mathrm{s}]$ \\
\hline$R_{x}$-axis max acceleration & $A_{x \max }$ & $2.0\left[\mathrm{~m} / \mathrm{s}^{2}\right]$ \\
\hline$R_{y}$-axis gain & $K_{y m}$ & $0.083[\mathrm{~m} / \mathrm{sV}]$ \\
\hline$R_{y}$-axis time constant & $T_{y m}$ & $0.006[\mathrm{~s}]$ \\
\hline$R_{y}$-axis max velocity & $V_{y \max }$ & $0.5[\mathrm{~m} / \mathrm{s}]$ \\
\hline$R_{y}$-axis max acceleration & $A_{y \max }$ & $1.0\left[\mathrm{~m} / \mathrm{s}^{2}\right]$ \\
\hline$R_{z}$-axis gain & $K_{z m}$ & $0.083[\mathrm{~m} / \mathrm{sV}]$ \\
\hline$R_{z}$-axis time constant & $T_{z m}$ & $0.007[\mathrm{~s}]$ \\
\hline$R_{z}$-axis max velocity & $V_{z \max }$ & $0.5[\mathrm{~m} / \mathrm{s}]$ \\
\hline$R_{z}$-axis max acceleration & $A_{z \max }$ & $1.0\left[\mathrm{~m} / \mathrm{s}^{2}\right]$ \\
\hline$R_{\theta}$-axis gain & $K_{t m}$ & $24.6[\mathrm{deg} / \mathrm{sV}]$ \\
\hline$R_{\theta}$-axis time constant & $T_{t m}$ & $0.006[\mathrm{~s}]$ \\
\hline$R_{\theta}$-axis max velocity & $V_{t \max }$ & $150[\mathrm{deg} / \mathrm{s}]$ \\
\hline$R_{\theta}$-axis max acceleration & $A_{t \max }$ & $1500\left[\mathrm{deg} / \mathrm{s}^{2}\right]$ \\
\hline$M$-axis gain & $K_{\operatorname{mm}}$ & $0.167[\mathrm{~m} / \mathrm{sV}]$ \\
\hline$M$-axis time constant & $T_{\operatorname{mm}}$ & $0.005[\mathrm{~s}]$ \\
\hline$M$-axis max velocity & $V_{\operatorname{mmax}}$ & $0.5[\mathrm{~m} / \mathrm{s}]$ \\
\hline$M$-axis max acceleration & $A_{\operatorname{mmax}}$ & $1.0\left[\mathrm{~m} / \mathrm{s}^{2}\right]$ \\
\hline & &
\end{tabular}

In previous paper, a fan-type ladle had been used as in Fig. 4(a) (Y. Sugimoto and K. Terashima, 2002). The surface area $A_{L f}$ of liquid in the fantype ladle is constant while the ladle is tilted. Therefore, it can be considered that the pouring flow rate $q_{f}(t)$ is constant when a ladle is rotated at a constant angular velocity. Hence, the flow rate model can be described as follows:

$$
\frac{d q_{f}(t)}{d t}=-\frac{1}{T_{f}} q_{f}(t)+\frac{K_{f}}{T_{f}} \omega(t)
$$

where $K_{f}\left[\mathrm{~m}^{3} / \mathrm{rad}\right]$ and $T_{f}[\mathrm{~s}]$ are the gain and the time constant of the flow rate model.

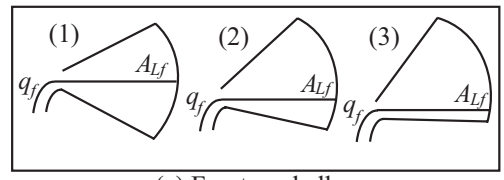

(a) Fan-type ladle

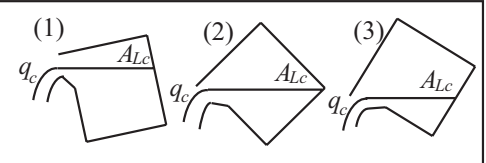

(b) Cylindrical ladle

Fig. 4. Flow characteristics of two type ladles

However, in this paper, a cylindrical ladle is used for the pouring. The surface area $A_{L c}$ of liquid in the cylindrical ladle is varying while the ladle is tilted as in Fig. 4(b). Therefore, the flow rate model of cylindrical ladle can not be represented as the linear model shown in Eq. (2). It can be considered that the flow rate $q_{c}$ depend on the tilting angle of cylindrical ladle. Hence, the flow rate model can be represented as LPV model, which gain and time constant are varied by tilting angle, as follows:

$$
\frac{d q_{c}(t)}{d t}=-\frac{1}{T_{c f}(\theta)} q_{c}(t)+\frac{K_{c f}(\theta)}{T_{c f}(\theta)} \omega(t)
$$

where $K_{c f}(\theta)\left[\mathrm{m}^{3} / \mathrm{rad}\right]$ and $T_{c f}(\theta)[\mathrm{s}]$ are the gain and time constant of flow rate model. $\theta$ is the tilting angle.

In order to identify these parameters by the experiment, the step input is given to the motor on $R_{\theta^{-}}$ axis as the ladle is tilted to $3[\mathrm{deg}]$ from each tilting angle, and then, overflow are obtained. Overflow obtained from calculation of Eq. (2) are fitted to the overflow obtained by experiment. Identified gain $K_{c f}(\theta)$ and time constant $T_{c f}(\theta)$ are shown in Fig. 5. In Fig. 5, until the tilting angle becomes 45 [deg] such that the ladle's condition is shown at (2) in Fig. 4(b), the flow rate gain is increased. After passing $45[\mathrm{deg}]$, the gain is decreased, since the surface area $A_{L c}$ of liquid in the cylindrical ladle becomes small. Therefore, it can be considered that identification of parameters is appropriate.
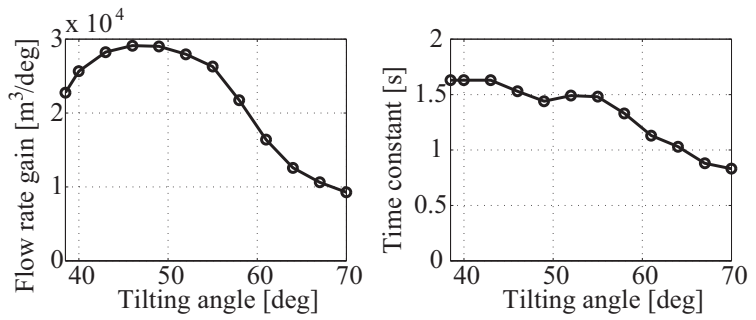

Fig. 5. Gain and time constant for tilting angle 
The liquid level model is built as shown in Fig. 3. So it is built by considering a mass balance, Bernoulli's theorem, and continuous equation(Y. Sugimoto and K. Terashima, 2002) as follows.

$$
\begin{aligned}
\frac{d h_{c}(t)}{d t}= & \frac{1}{A_{C}} q_{c}(t) \\
& -c \frac{A_{G}}{A_{C}} \sqrt{2 g\left(h_{c}(t)+h_{1}-h_{s}(t)\right)} \\
\frac{d h_{m}(t)}{d t}= & c \frac{A_{G}}{A_{M}} \sqrt{2 g\left(h_{c}(t)+h_{1}-h_{s}(t)\right)}
\end{aligned}
$$

where $c$ is the flow coefficient and $g$ is gravitational acceleration. In this paper, the flow coefficient $c$ is 0.668. And, if $h_{s}(t)$ is under $0[\mathrm{~m}], h_{s}(t)$ is shown as $0[\mathrm{~m}]$.

Finally, a model from control input $u_{t}(t)$ to liquid level $h_{c}(t)$ in sprue cup is as follows:

$$
P(s)=P_{m t}(s) P_{f}(s) P_{h}(s)
$$

\section{FF CONTROL OF LIQUID LEVEL}

FF control can achieve a high degree of accuracy when it is used in the appropriate situation. In order to shorten the cycle time and maintain steady control such as a constant level in a sprue cup, it is strongly required to have FF control when the flow pattern is specified beforehand. We call an ideal flow rate of a pouring pattern. Since the paper has a limitation of pages, the ideal flow rate is briefly described in this paper. The detail of ideal flow rate is referred in previous paper(Y. Sugimoto and K. Terashima, 2002). The ideal flow rate used in this paper is shown in Fig. 6. In Fig. 6 , the rising part of ideal flow rate is represented as combination of sinusoidal curves to avoid the rapid change of motor input(Y. Sugimoto and K. Terashima, 2002). And, the parameter is adjusted such as achieving quickly the liquid level to the target level $(0.06[\mathrm{~m}])$.

In equilibrium part, the flow rate $q_{r e f_{e}}(t)$ at the target liquid level is obtained as follows:
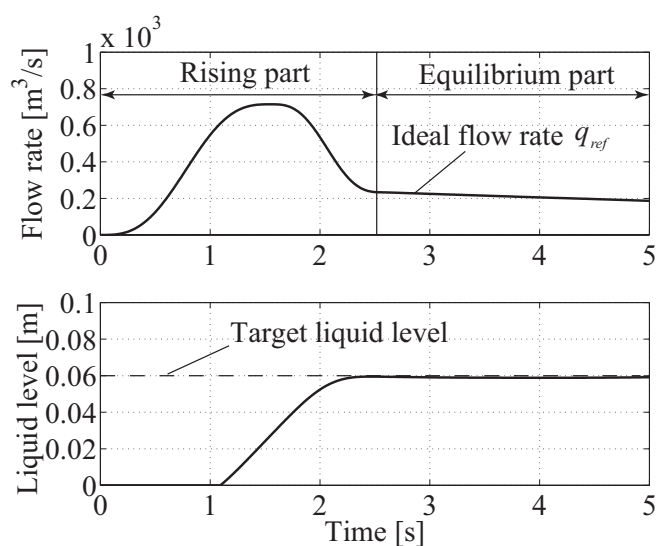

Fig. 6. Ideal flow rate and liquid level in sprue cup

$$
q_{r e f_{e}}(t)=c A_{G} \sqrt{2 g\left(h_{r e f}+h_{1}-h_{m}(t)\right)}
$$

, where $h_{\text {ref }}$ is target liquid level in the sprue cup.

Control input $u_{t}(t)$ during tilting of a ladle can be calculated by inverse model as shown in Fig. 7 .

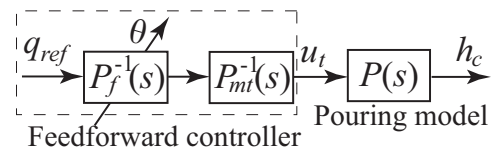

Fig. 7. FF controller for pouring process

As verification of FF control for pouring model using cylindrical ladle, the experimental results is shown in Fig. 8. In the experiment, the pouring robot pours into three molds as shown in Fig. 2. Fig. 8(a) shows control inputs that are obtained by calculating the inverse model as shown in Fig. 7. And, Fig. 8(b)-(d) show liquid levels in sprue cups of each molds. As a result, since the liquid levels of the experiment result are corresponded to that of simulation result, the liquid levels can be achieved quickly to target liquid level by the proposed method.
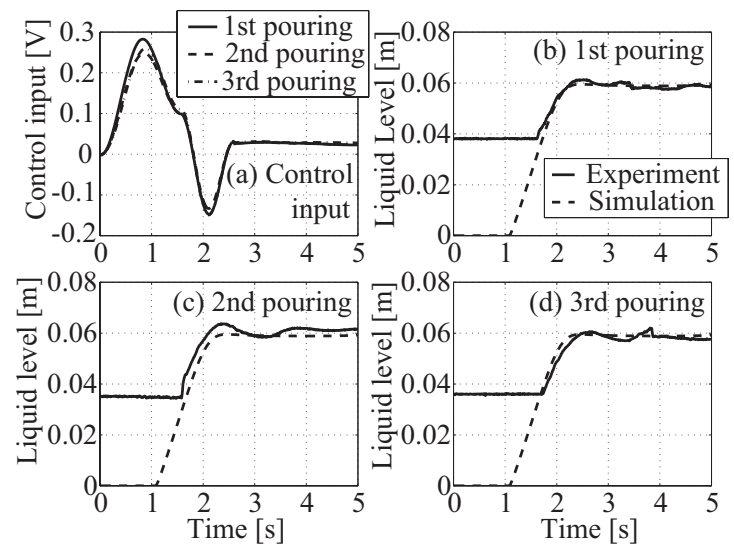

Fig. 8. Experimental results of pouring control

\section{TRACKING CONTROL WITH CONSIDERING SLOSHING SUPPRESSION}

Tracking control with considering sloshing suppression, which is needed to self-transfer-type automatic pouring robot, is built by using $R_{x^{-}}$ and $M$-axes as shown in Fig. 2. And, the control system is shown in Fig. 9. This system has been published by the author's previous paper(Y. Noda and K. Terashima, 2003). In order to track quickly to the moving mold, the inverse model of the motor is applied to FF controller in the pouring robot. And, in order to suppress the sloshing, FeedBack(FB) controllers $K_{m}$ and $K_{x}$ are designed by Hybrid Shape Approach. Moreover, the systems of mold line and pouring robot are connected while suppressing the sloshing by using Time Varying Gain Filter $K_{v}(\mathrm{Y}$. Noda and K. Terashima, 2003). However, the sloshing 


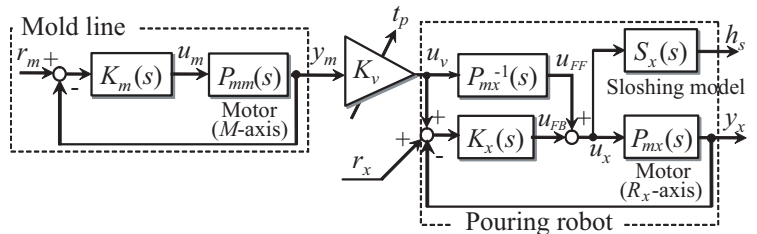

Fig. 9. Control system for tracking

caused by tracking during the pouring motion has not been considered in the previous paper. By the pouring motion, the natural frequency of the sloshing is varied, because the liquid quantity is decreased with the increase of pouring time. Therefore, the sloshing is analyzed by using Short Time Fourier Transform in order to conduct the frequency analysis in the non-stationary process, and then, the feedback controllers are rebuilt by Hybrid Shape Approach(K. Yano and K. Terashima, 2001).

\subsection{Analysis of sloshing}

Short Time Fourier Transform is shown as follows:

$$
S p(t, f)=\left|\int_{-\infty}^{\infty} h_{s}(t) w(\tau) e^{-j 2 \pi f \tau} d \tau\right|^{2}
$$

where $w(t)$ and $h_{s}(t)$ are window functions and sloshing data. In this paper, the number of window size is 256 . The sloshing data is obtained by transferring the ladle on $R_{x}$-axis while pouring. FB system with $\mathrm{P}$ control is used to the transfer system in order to cause sloshing. By the experiment, the position of ladle on $R_{x}$-axis, the tilting angle on $R_{\theta}$-axis, and the sloshing in the ladle are obtained as shown in Fig. 10. Fig. 10 shows the experimental results for 1st pouring. At first, the sloshing is caused by transferring the ladle on $R_{x}$-axis. Then, the ladle is tilted as the pouring motion. In the result of sloshing, the sloshing data has been compensated for decreased the liquid level by pouring. The energy contour of the sloshing analyzed by Short Time Fourier Transform is shown in Fig. 11. It is shown that region with the dense contour has higher energy than the other region.
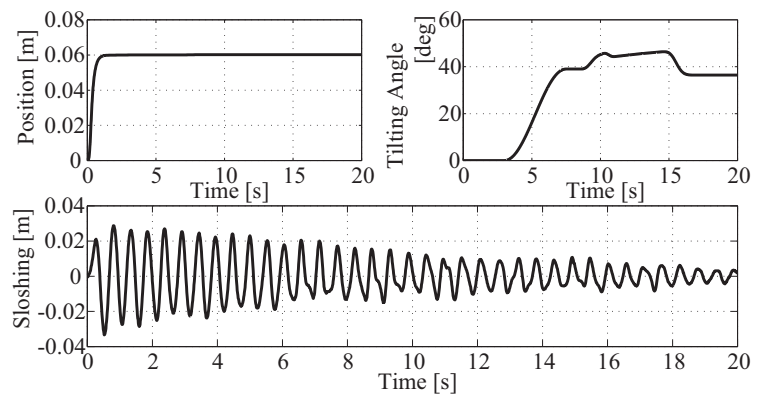

Fig. 10. Experimental results of positions and sloshing for sloshing analysis (1st pouring)

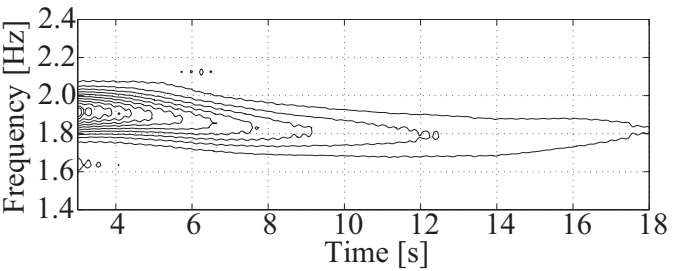

Fig. 11. Energy contour of sloshing (1st pouring)
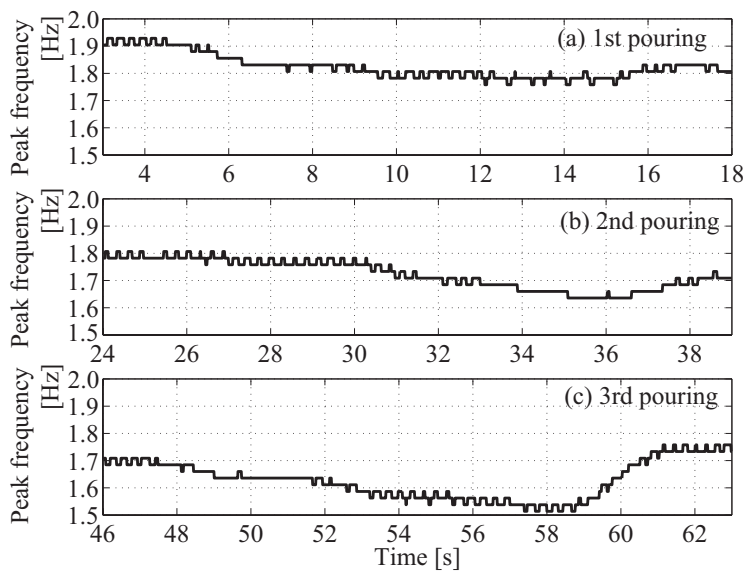

Fig. 12. Peak frequency obtained from Short Time Fourier Transform

The energy peaks at each time are plotted to Fig. 12. In Fig. 12, energy peaks at each time are shown for the pouring motions from 1st to 3rd. Energy peaks of 2 nd and 3rd pouring are obtained by similar method with 1st pouring. The peak frequency from $3[\mathrm{~s}]$ to $4[\mathrm{~s}]$ in Fig. $12(\mathrm{a})$ is $1.93[\mathrm{~Hz}]$. Since the initial liquid level is $0.16[\mathrm{~m}]$, the natural frequency of sloshing is obtained as $1.93[\mathrm{~Hz}]$ by calculating theoretically(K. Yano and K. Terashima, 2001). Therefore, the peak frequency from Short Time Fourier Transform has well identified the natural frequency of sloshing. As a result, it can be seen that the natural frequency of sloshing is varied from $1.93[\mathrm{~Hz}]$ to $1.51[\mathrm{~Hz}]$ by three times pouring.

\subsection{Design of feedback controllers}

Feedback controllers $K_{m}$ and $K_{x}$ are designed by using Hybrid Shape Approach(K. Yano and K. Terashima, 2001). The controllers are formulated by using controller elements as follows.

$$
\begin{array}{r}
K_{i}(s)=\frac{K_{p i}}{T_{l i} s+1} \prod_{k=1}^{2} \frac{s^{2}+2 \zeta \omega_{n k} s+\omega_{n k}^{2}}{s^{2}+\zeta \omega_{n k} s+\omega_{n k}} \\
(i=m, x)
\end{array}
$$

These controllers consist of 4 controller elements, which are proportional $(P)$ gain, low-pass filter, and 2 notch filters. In order to reduce the influences of higher-mode sloshing and noise, a lowpass filter, which makes the controller low gain at a high frequency domain, is selected. 2 notch 

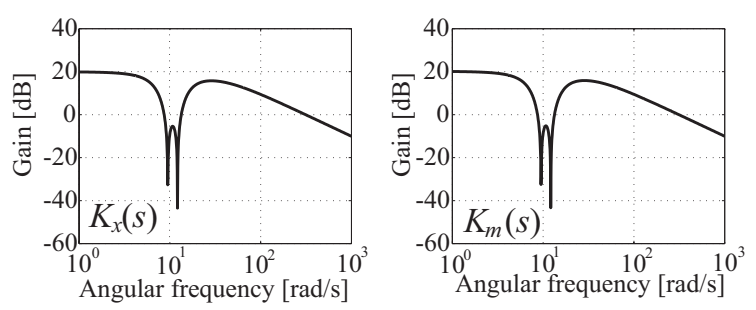

Fig. 13. Gain diagrams of FB controllers

filters are selected in order to suppress sloshing. Resonance frequencies $\omega_{n k}$ of the notch filters are corresponded to natural frequencies of sloshing as $w_{n 1}=12.17[\mathrm{rad} / \mathrm{s}](1.93[\mathrm{~Hz}])$ and $w_{n 2}=9.49[\mathrm{rad} / \mathrm{s}]$ $(1.51[\mathrm{~Hz}])$ are obtained in previous section. $K_{p i}$ and $T_{l i}$ are reasonably determined by solving the optimization problem, which the settling time is minimized and control specifications(limitations of control input and so on) are satisfied.

Fig. 13 shows the gain diagrams of the controllers obtained by above process. Since the gains of both controllers are reduced on band width from $\omega_{n 1}$ to $\omega_{n 2}$, the sloshing can be damped even if the ladle is transferred with pouring motion.

\subsection{Experimental result}

As the verification of sloshing suppression control, the experiment of tracking to moving molds with pouring motion is accomplished by self-transfertype automatic pouring robot as shown in Fig. 2. Fig. 14 shows the experimental results of the positions of pouring robot and molds, control input on $R_{x}$-axis, and tilting angle of ladle.

In the experiment, the pouring robot tracks to 3 target molds. At first, the molds are transferred as a sinusoidal curve. After $4.5[\mathrm{~s}]$, the pouring robot starts to track to 1st mold, and after tracking, the ladle is tilted for pouring liquid into the mold. Pouring into 2nd and 3rd mold are accomplished in the same way. Fig. 15 shows the experimental results of sloshing in a cylindrical ladle caused by

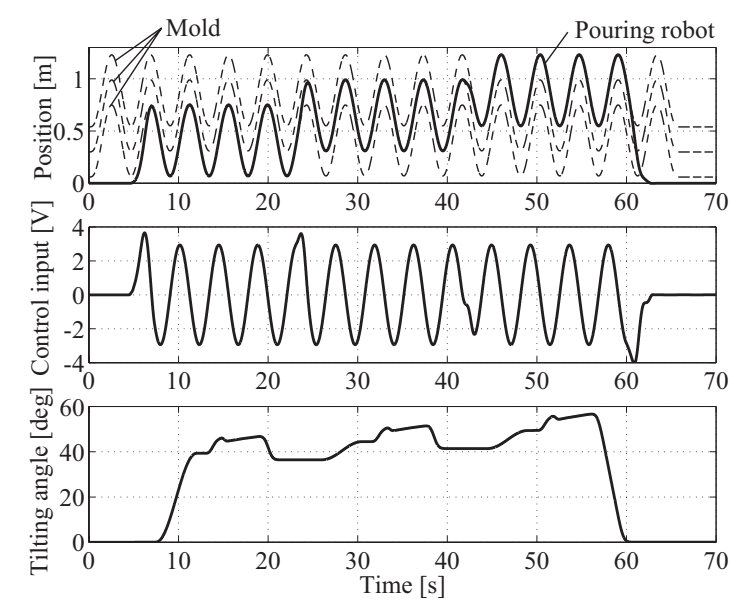

Fig. 14. Experimental results of self-transfer-type automatic pouring robot

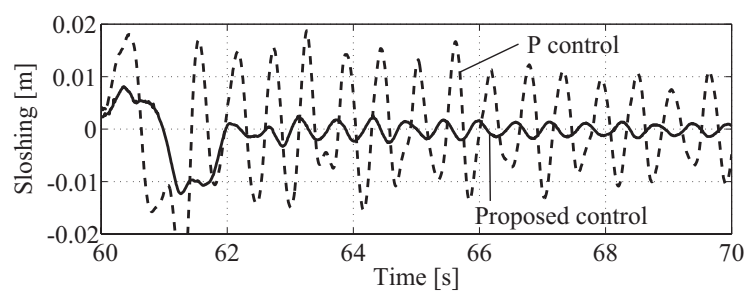

Fig. 15. Experimental results of sloshing in a ladle

the motion as shown in Fig. 14. As comparison of the proposed control, a control system with $P$ control as FB controller in Fig. 9 is used. In order to compare the residual vibrations, the sloshing after finishing the tracking and pouring motion have been shown. The sloshing by using $P$ control is generated. On the other hand, the sloshing by proposed control is under $0.002[\mathrm{~m}]$. Hence, the self-transfer-type automatic pouring robot considering with sloshing suppression was well realized by the proposed method. Furthermore, during synchronous transfer of ladle and mold, liquid of a ladle was exactly poured into a mold, and liquid level in a sprue cup of a mold has been well achieved at the constant level.

\section{CONCLUSION}

This paper has presented a development of selfTransfer-type automatic pouring robot using cylindrical ladle and considering sloshing suppression. To the pouring motion to cylindrical ladle, LPV model was built as the flow rate model. To the sloshing suppression control for tracking to the moving mold with pouring motion, the varying natural frequency was identified by using Short Time Fourier Transform, and the sloshing could be damped by using Hybrid Shape Approach. Through various experimental results, it was demonstrated that the proposed control well satisfied the present requirements.

\section{REFERENCES}

K. Yano, T. Toda, and K. Terashima (2001). Sloshing Suppression Control of Automatic Pouring Robot by Hybrid Shape Approach, Proc. of the 40th IEEE Conference on Decision and Control, pp. 1328-1333.

Y. Noda, K. Yano, and K. Terashima (2003). Detection and Tracking Control to Unknown Moving Object Considering Sloshing-Suppression in Pouring Robot, Proc. of the 23rd IASTED International Conference MODELLING, IDENTIFICATION, AND CONTROL, pp. 345-350.

Y. Sugimoto, K. Yano, and K. Terashima (2002). Liquid Level Control of Automatic Pouring Robot by Two-Degree-Of-Freedom Control, IFAC 15th Triennial World Congress, pp. 1102.pdf(1)-(6). 